\title{
Characterization of $\mathrm{MoS}_{2}$ Nanorods by Electron Microscopy
}

\section{Anthony Salazar ${ }^{1}$, Diana Sanchez ${ }^{1}$, Christian Kisielowski ${ }^{2}$, Jungiao Wu ${ }^{1}$, Oscar Dubon ${ }^{1}$ and Hector Calderon $^{3}$}

${ }^{1}$ University of California at Berkeley, Berkeley, California, United States, ${ }^{2}$ Lawrene Berkeley National Laboratory, The Molecular Foundry and Joint center for Artifical Photosynthesis, University of California, Berkeley, United States, ${ }^{3}$ Instituto Politecnico Nacional, Zacatenco, Mexico

Semiconducting nanowires demonstrate many useful properties due to quantum confinement, and have applications ranging from electronic and optoelectronic devices to chemical and biological sensors [1]. A reliable method to grow nanowires is the vapor liquid solid technique, in which a liquid metal catalyst is super saturated by the precursor elements until the nanowire precipitates out of the catalyst. Layered semiconductors such as transition metal dichalcogenides have natural anisotropy and using quantum confinement the band gap energies can be tuned. MoS2 is a layered semiconductor in the transition metal dichalcogenide family. It has a band gap in the visible region, and has demonstrated good on/off ratio and mobility when used to make transistors. Reports on MoS2 1-D nanostructures are primarily of nanotubes and nanoribbons which are fixed to the growth substrate [2]. By using a tube furnace, the vapor liquid solid technique is applied to grow free standing MoS2 nanowires. To understand the influence of the metal catalyst orientation on the growth of $\mathrm{MoS} 2$ nanowires low does electron microscopy has been employed Electron microscopy has been performed in a low dose condition in order to image the genuine structure of the samples by using focal series and exit wave reconstruction in transmission electron microscopy. The microscope in use has been the TEAM 1 at $300 \mathrm{kV}$ at the MF-LBNL [3]. The focal series consisted of 50 images at different focal settings. The software package MackTempas X has been used to recover phase and amplitude images with atomic resolution via exit wave reconstruction (EWR). The electron dose rate has been of approximately 10 e/Å2s. Figure 1 shows a panoramic view of a MoS 2 nanorod and the supporting gold seed. The growth mechanism minimizes the contact area due to reduction of the total interfacial surface area and the subsequent growth of the nanorod includes an enlargement of the total diameter. The rod becomes stable and the growth proceeds in a single direction along the main axis. Figure 2 shows a phase image from a section of the area in Figure 1. Here the atomic distribution corresponding to $\mathrm{MoS} 2$ can be identified. The Au lattice is also imaged. The corresponding diffraction pattern is included, the lattice reflections show the presence of internal strains. The nanorod atomic distribution includes stacking faults with their correspondence plane along the axis of the nanorod, additionally some Au contamination near the contact surface can be seen. Otherwise as the rod increases in length, the atomic distribution can be identified as that of $\mathrm{MoS} 2$, although some variations in crystalline structure develop as growth proceeds. 


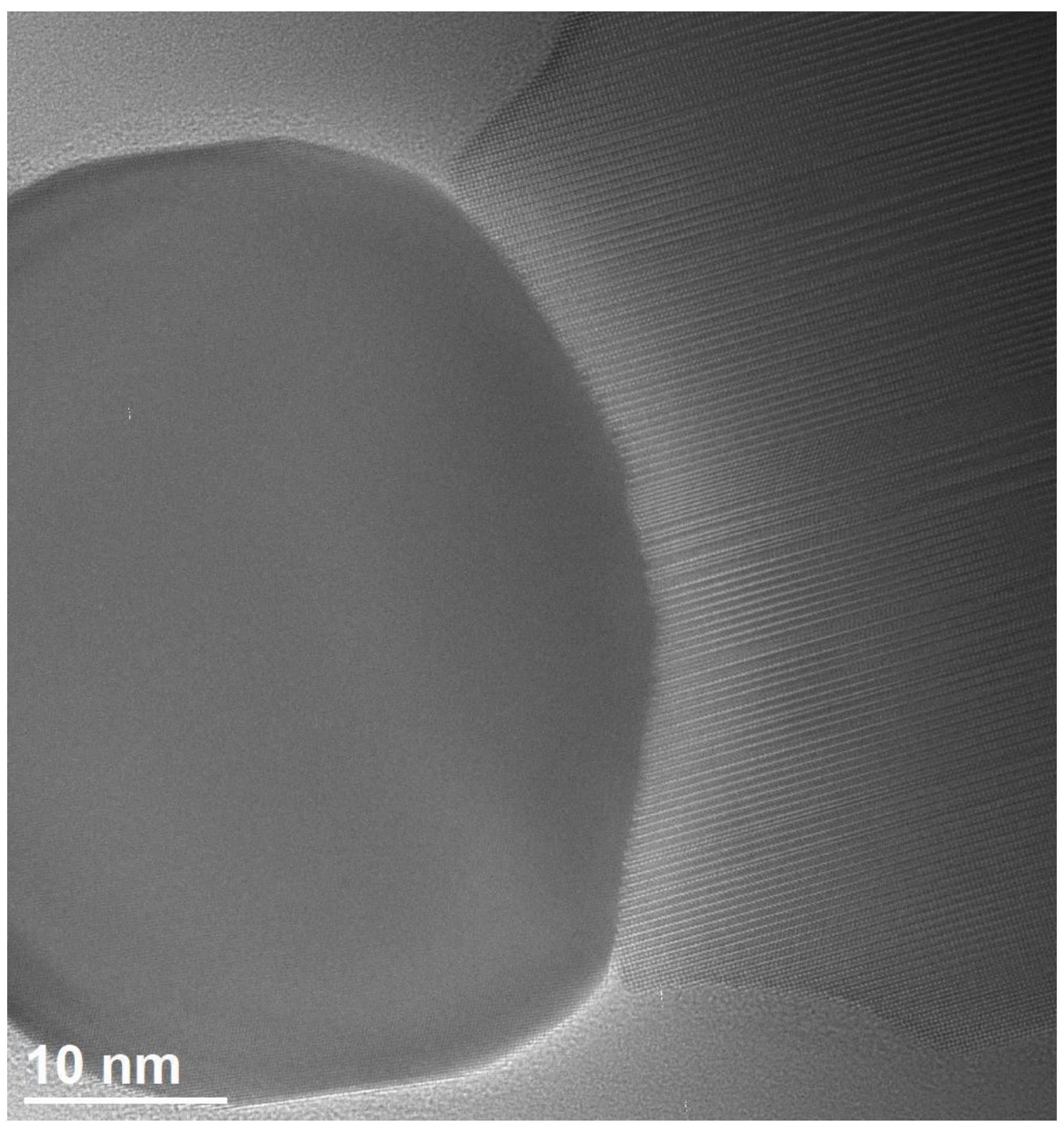

Figure 1. Figure 1. TEM image of the Au seed, the MoS2 nanorod and the surface interface that develops during the growing stage. 


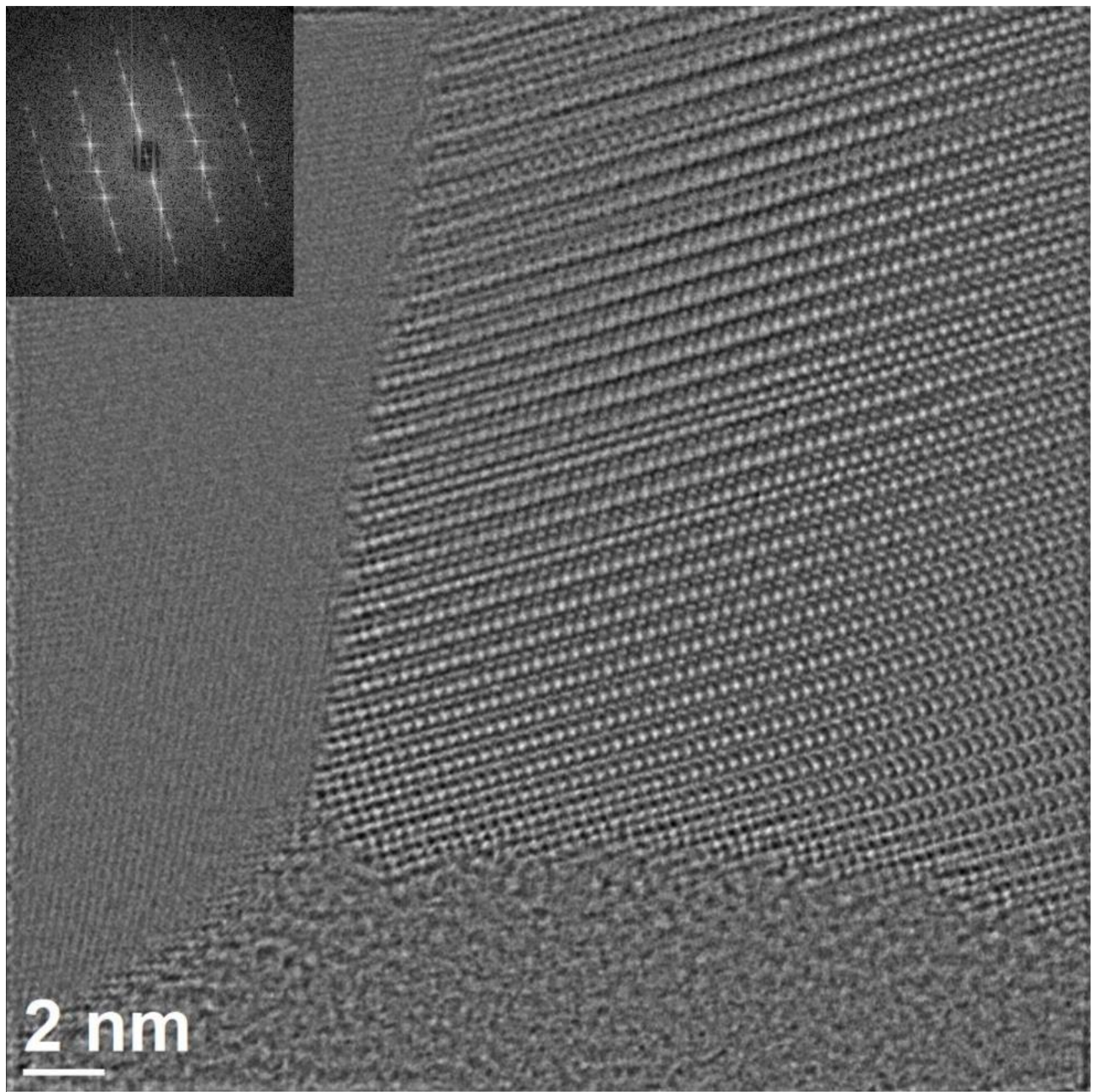

Figure 2. Figure 2. Phase image of the interface between the Au seed and the MoS2 nanorod. The phase image is derived from an exit wave reconstruction with a focal series consisting of 50 images.

References

1. E. Sutter, P. Sutter. (2018). 1D Wires of 2D Layered Materials: Germanium Sulfide Nanowires as Efficient Light Emitters. ACS Applied Nano Materials, 1(3), 1042-1049. https://doi.org/10.1021/acsanm.7b00053.

2. W. Mengting, "Growth of MoS2 Nanowires by Catalytic Chemical Vapor Deposition." Doctoral Dissertation, Hokkaido University(2018). DOI:10.14943/doctoral.k13226.

3. Work at the Molecular Foundry was supported by the Office of Science, Office of Basic Energy Sciences, of the U.S. Department of Energy under Contract No. DE-AC02-05CH11231. 